\title{
AVALIAÇÃO COMPARATIVA DA METODOLOGIA DE DETERMINAÇÃ̃ DA ÁREA DE OLHO DE LOMBO EM SUÍNOS (Sus domesticus)
}

\author{
Maurício de Paula Ferreira Teixeira ${ }^{1}$, Juliana Fortes Vilarinho Braga², Manoel Henrique Klein \\ JÚNIOR ${ }^{3}$, MÁRvio Lobão TEIXeIRA ABREU ${ }^{4}$
}

\begin{abstract}
1 - Doutorando em Zootecnia, Universidade Federal de Minas Gerais - teixeirampf@hotmail.com 2 - Médica Veterinária, Mestre em Ciência Animal, Universidade Federal do Piauí

3 - Professor Adjunto, Departamento de Morfofisiologia Veterinária, Universidade Federal do Piauí

4 - Professor Associado, Departamento de Zootecnia, Universidade Federal do Piauí
\end{abstract}

\section{RESUMO}

Em estudos que envolvem as características de carcaça realizados no Departamento de Zootecnia do Centro de Ciências Agrárias da Universidade Federal do Piauí, medidas lineares da área de olho de lombo (AOL) e da área da gordura subcutânea (AGSC) vêm sendo mensuradas, com leituras realizadas com a contagem em $\mathrm{cm}^{2}$ pelo método do plástico quadriculado. Com a finalidade de testar essa metodologia amplamente empregada, o presente trabalho teve como objetivo realizar um estudo comparativo com três outros procedimentos de maior ou menor precisão no sentido de verificar a acurácia do método tradicional. Foi utilizada a análise de variância (ANOVA) em esquema fatorial $2 \times 4$ para estimar os efeitos dos dois padrões genéticos e dos quatro métodos de deterninação (gabarito, autoCad, planímetro e pesagem) sobre as AOL e AGSC. Não houve diferença entre as quatro metodologias e o método do gabarito mostrou-se de maior facilidade de execução, praticidade e confiabilidade para utilização em pesquisa.

PALAVRAS-CHAVES: Área de olho de lombo; gordura subcutânea; mensuração.

\section{COMPARATIVE EVALUATION OF THE METHODOLOGY FOR SWINE ( $\mathrm{Sus}$ domesticus) RIB EYE AREA DETERMINATION}

In studies involving the carcass characteristics conducted at the Department of Animal Science of the Federal University of Piauí, the linear measurements of rib eye area (REA) and subcutaneous fat area (SFA) have been carried out by the plastic grid method, in which readings are made by counting in $\mathrm{cm}^{2}$. In order to test this widely used approach, this study aimed at conducting a comparison among three other more or less precise procedures in order to verify the traditional method accuracy. To estimate the effect of both genetic patterns and the four determination methods (Feedback, AutoCAD, Planimeter, and Weighing) on REA and SFA, analysis of variance (ANOVA) was used in $2 \times 4$ factorial arrangement. There was no difference among the four 
methodologies and the feedback method shows easier implementation, more practicality and reliability to be

used in researches.

KEYWORDS: Measurement; rib eye area; subcutaneous fat.

\section{INTRODUÇÃO}

Devido às exigências atuais e futuras do mercado de carne brasileiro, há necessidade de se produzir animais que tenham boa qualidade de carcaça, com máximo de cortes comercializáveis e boa cobertura de gordura. Nesse sentido, a avaliação da qualidade de carcaça de animais é de fundamental importância, podendo trazer diferenciais de remuneração aos criadores. $\mathrm{O}$ crescimento anual da produção de animais precoces, através de programas de cruzamentos industriais e do uso de raças geneticamente superiores, vem gerando uma série de estudos e o desenvolvimento de novas técnicas de manipulação e predição da qualidade da carcaça e da carne (GUIDONI, 2000).

Pesquisas mostram que quanto maior a área de olho de lombo (AOL), maior será o rendimento da carcaça em cortes comercializáveis e que a espessura de gordura subcutânea (EGS) é de suma importância para os atributos qualitativos da carcaça (JORGE et. al., 1999; SILVA \& PIRES, 2000; SIQUEIRA et al., 2001; YOKOO et al., 2009). Segundo WILSON (1995), AOL e EGS são determinações altamente correlacionadas com cortes desossados da carcaça, sendo a EGS correlacionada inversamente.

As medidas realizadas na carcaça permitem comparações entre tipos raciais, peso e idades de abate, sistemas de alimentação e, também, o estabelecimento de correlações com outras medidas ou com os tecidos constituintes da carcaça, possibilitando a estimação de suas características físicas, evitando, dessa forma, o oneroso processo de dissecação de carcaça (SILVA \& PIRES, 2000).

A avaliação da área de olho de lombo (AOL) e da espessura de gordura subcutânea, obtida entre a $12^{\mathrm{a}}$ e $13^{\mathrm{a}}$ vértebra torácica, tem sido amplamente aceita e utilizada como indicador da composição da carcaça (LUCHIARI FILHO, 2000), evitando-se outras ações trabalhosas e demoradas. Assim, reduz-se o número de medidas, eliminando-se, inclusive, aquelas que menores contribuições trazem para a avaliação da qualidade das carcaças.

O método tradicional para a obtenção da AOL tem sido realizado com o auxílio de um gaba- rito plástico quadriculado ou com papel vegetal, utilizado em bovinos (RIBEIRO et al., 2002; ROMA JÚNIOR, 2004; PINHEIRO, 2007), suínos (FIALHO et al., 1998; DUTRA JR et al., 2001; OLIVEIRA et al., 2003; FREITAS et al., 2004), ovinos (CUNHA et al, 2000; CUNHA et al., 2001; VELOSO et al., 2004; MENEZES et al., 2008) e até em animais silvestres como a capivara (PINHEIRO et al., 2007),

Em estudos que envolvem as características de carcaça realizados no Departamento de Zootecnia do Centro de Ciências Agrárias da Universidade Federal do Piauí, medidas lineares da AOL e da área da gordura subcutânea (AGSC) vêm sendo mensuradas, com leituras realizadas com a contagem em $\mathrm{cm}^{2}$ pelo método do plástico quadriculado. Com o presente trabalho, objetivou-se comparar essa metodologia com três outras de maior e menor precisão no sentido de verificar a acurácia do método tradicional.

\section{MATERIAL E MÉTODOS}

Para as determinações das AOL e AGSC foram utilizados cortes de suínos. As amostras do corte técnico referentes ao lombo foram obtidas de 40 animais, sendo 20 híbridos comerciais e 20 nativos. Todos os animais foram produzidos na forma de ciclo completo no Departamento de Zootecnia (DZO), do Centro de Ciências Agrárias (CCA), da Universidade Federal do Piauí (UFPI). Os suínos foram criados em baias coletivas de quatro $\mathrm{m}^{2}$, submetidos a uma dieta balanceada, formuladas de modo a atender às exigências dos animais, e abatidos entre cinco a seis meses de idade. Para a coleta das amostras, a carcaça ainda quente foi dividida com ajuda de serra elétrica. O lombo foi retirado da meia carcaça esquerda pela separação do dianteiro entre a $5^{\mathrm{a}}$ e a $6^{\mathrm{a}}$ vértebras torácicas e última lombar e ainda da costela com barriga por serragem à distância de, aproximadamente, 2 $\mathrm{cm}$ a partir do início das costelas.

Após o acondicionamento individual em sacos de polietileno com suas devidas identificações, as peças foram imediatamente colocadas em freezer doméstico, no Núcleo de Estudos Pesquisa e Processamento de Alimentos (NUEPPA) do CCA, da UFPI, 
para posterior análise.

Nas peças congeladas foram feitos dois cortes transversais, por meio de serra elétrica, sendo o primeiro entre a $12^{\mathrm{a}}$ e a $13^{\mathrm{a}}$ vértebras torácicas e outro entre a $13^{\mathrm{a}}$ vértebra torácica e a vértebra subsequente. Esse procedimento foi realizado para diminuir a espessura do corte e facilitar o manuseio da peça proporcionando uma melhor aderência em uma superfície plana. A região usada para mensurar a área do olho de lombo (AOL) e a área da gordura subcutânea (AGSC) foi a da superfície da porção cranial da $13^{\mathrm{a}}$ vértebra torácica.

Após os cortes, a área a ser mensurada foi limpa, colocando-se sobre ela papel vegetal, tamanho 20x15 cm, no qual foi desenhado com lápis o contorno da porção muscular e da cobertura de gordura com a pele. A AOL se limitou apenas ao músculo longissimus dorsi, de acordo com a metodologia de avaliação de carcaça da suína padronizada pela Associação Brasileira dos Criadores de Suínos (ABCS, 1973). Para delimitação da AGSC a ser determinada, traçou-se uma linha tangenciando as extremidades da região do músculo longissimus dorsi.

Para a determinação da AOL e da AGSC foram utilizados, além da metodologia tradicional, outros três métodos descritos a seguir, todos tendo como base o desenho obtido em papel vegetal.

a) Método tradicional (gabarito), também conhecido como gabarito plástico quadriculado.

A partir de uma folha de papel milimetrado dividida em quadrados de um e de $0,25 \mathrm{~cm}^{2}$ foram confeccionadas 40 cópias em folha plástica transparente, obtendo-se desta forma o gabarito. Esse era então posicionado sobre os contornos originais no papel vegetal e o número de quadrados de um $\mathrm{cm}^{2}$ eram inicialmente marcados. Foram também quantificadas regiões correspondentes a no máximo a metade dos quadrados de $0,25 \mathrm{~cm}^{2}$. Realizou-se a soma dos quadrados de um, $0,25 \mathrm{~cm}^{2}$ e da metade deste, obtendo-se, assim, a área total. Esse procedimento foi realizado tanto para a região muscular quanto para a da gordura subcutânea.

b) Utilização do software AutoCAD.

Após prévia digitalização dos desenhos, os arquivos foram inseridos no programa AutoCAD versão 2007, utilizado em cálculos de engenharia civil. Em cada figura era inicialmente determinada a escala e feito o contorno de cada região individualmente com identificação por hachuras. Em seguida, era ob- tida a área desejada juntamente com o perímetro. Esses cálculos foram realizados na Divisão de Projetos e Obras da UFPI.

c) Utilização de planímetro analítico (planímetro)

Esta determinação foi efetuada em aparelho utilizado para medir áreas planas de formatos irregulares, em cartografia, existente no Departamento de Engenharia de Agrimensura do Centro de Tecnologia da UFPI. A medida foi obtida contornando-se diretamente no papel vegetal que continha o desenho original. $\mathrm{O}$ resultado era obtido a partir da média de três leituras multiplicada por fator de correção (US) para a obtenção do resultado nessa escala métrica.

d) Estimativa por pesagem dos contornos em papel padronizado (pesagem)

Utilizou-se folha de papel A4 $(21 \times 29,7=$ $623,7 \mathrm{~cm}^{2}$ ) na qual eram copiados por justaposição os contornos originais e então registrado o peso de cada folha em balança semi-analítica. Os desenhos referentes ao lombo e a gordura de cobertura eram então recortados e pesados. O cálculo foi efetuado com uso da regra de três, considerando o peso da folha íntegra com o desenho correspondendo à área total da folha $\left(623,7 \mathrm{~cm}^{2}\right)$. A partir daí, calculava-se a proporção do peso de cada região recortada correspondendo a AOL e AGSC (Figura 1).

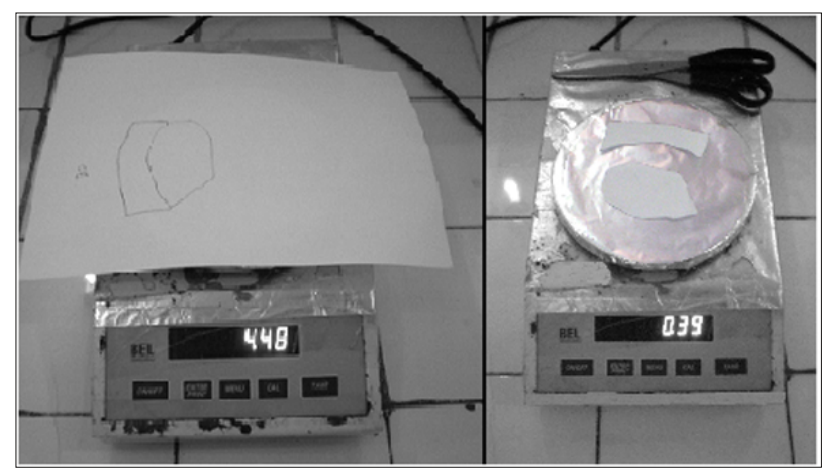

Figura 1. Estimativa por pesagem dos contornos em papel padronizado para o cálculo das AOL e AGSC.

Os dados foram submetidos à análise de variância e as médias comparadas pelo teste de StudentNewman-Keuls utilizando-se o Statistical Analysis System (SAS, 1986).

\section{RESULTADOS E DISCUSSÃO}

Os resultados obtidos estão apresentados na Tabela 1. Não foi verificado efeito de interação entre os dois padrões genéticos e os quatro métodos de determinação para os cálculos das AOL e AGSC. 
Tabela 1. Médias e coeficientes de variação (CV) sobre a AOL e AGSC em $\mathrm{cm}^{2}$ do corte comercial do lombo de suínos melhorados e nativos determinados por quatro métodos

\begin{tabular}{|c|c|c|}
\hline $\begin{array}{c}\text { Métodos de } \\
\text { Determinação }\end{array}$ & \multicolumn{2}{|c|}{ Padrão genético } \\
\hline & Nativos & Melhorados \\
\hline & \multicolumn{2}{|c|}{ AGSC } \\
\hline Gabarito & $12,83 \pm 2.91^{\mathrm{a}}$ & $18,38 \pm 4.13^{b}$ \\
\hline AutoCAD & $12,75 \pm 2.62^{\mathrm{a}}$ & $18,62 \pm 3.99^{\circ}$ \\
\hline Planimetro & $13,12 \pm 2.84^{\mathrm{a}}$ & $18,78 \pm 3.92^{\circ}$ \\
\hline Pesagem & $12,58 \pm 3.58^{\mathrm{a}}$ & $18,31 \pm 3.99^{b}$ \\
\hline Média & $12,82^{\mathrm{a}}$ & $18,52^{\circ}$ \\
\hline \multirow[t]{2}{*}{$\mathrm{CV}(\%)$} & \multicolumn{2}{|c|}{22,65} \\
\hline & \multicolumn{2}{|c|}{$\mathrm{AOL}$} \\
\hline Gabarito & $13,01 \pm 1.62^{\mathrm{a}}$ & $33,77 \pm 5.97^{b}$ \\
\hline AutoCAD & $12,90 \pm 1.60^{\mathrm{a}}$ & $35,14 \pm 8.05^{b}$ \\
\hline Planimetro & $13,18 \pm 1.52^{\mathrm{a}}$ & $33,48 \pm 5.75^{b}$ \\
\hline Pesagem & $13,32 \pm 1.78^{\mathrm{a}}$ & $32,76 \pm 5.33^{b}$ \\
\hline Média & $13,10^{\mathrm{a}}$ & $33,79^{b}$ \\
\hline $\mathrm{CV}(\%)$ & \multicolumn{2}{|c|}{19,83} \\
\hline
\end{tabular}

Médias seguidas pela mesma letra minúscula não diferem $(\mathrm{P}<0,05)$ em uma mesma coluna - Teste Student Newman Keuls.

Considerando não ter havido diferença entre os métodos, é interessante a avaliação das vantagens e desvantagens de cada metodologia no sentido de considerar a sua utilização visando principalmente a sua facilidade de execução.

Para a utilização do gabarito quadriculado há necessidade da folha quadriculada transparente e caneta apropriada. Apesar de relativa facilidade, devese ter cuidado na justaposição e fixação do desenho original para as contagens dos quadrados. O método é considerado prático, existindo a possibilidade de ser usado sem a necessidade do desenho, ou seja, diretamente no corte ou mesmo logo após a obtenção da região a ser medida, na própria câmara fria, sendo, portanto, o método de maior utilização, em variadas espécies produtoras de carne (FIALHO et al., 1998; CUNHA et al., 2000; CUNHA et al., 2001; DUTRA JR et al., 2001; RIBEIRO et al., 2002; OLIVEIRA et al., 2003; FREITAS et al., 2004; ROMA JÚNIOR, 2004; VELOSO et al., 2004; PINHEIRO et al., 2007; MENEZES et al., 2008), até mesmo para confirmar a utilização da ultra-sonografia (PERKINS et al., 1992; PINHEIRO, 2007).

Não foi possível obter citações referentes à utilização dos métodos utilizados para o estudo comparativo com o tradicional utilizando o gabarito plástico. Contudo, havendo a disponibilidade dos equipamentos necessários, qualquer um poderá ser utilizado. Constatou-se que em vários trabalhos consultados não havia descrição da metodologia utilizada para determinação da AOL.

A utilização de software (autoCAD) foi a principio o método considerado como a metodologia padrão por apresentar uma menor possibilidade de erro, com maior precisão. No entanto, após a digitalização, a imagem tem que ser redefinida pelo operador do programa, requerendo com isso certa habilidade no manuseio e a necessidade de algum tempo. Deve ser também levado em conta a necessidade da prévia digitalização das imagens em equipamento escaneador digital para inserção no programa. Contudo, é possível, com um mínimo de treinamento, manusear o programa sem dificuldades. Deve ser considerada a necessidade da aquisição do hardware e do software.

Com relação à utilização do planímetro analítico (planímetro), ele deve estar rigorosamente calibrado para determinação do fator de correção. Essa operação é feita uma única vez por uso, tendo que ser novamente ajustado caso vá ser utilizado em outra ocasião. No experimento, foi necessária a realização de três ajustes. Esse fator (US) deverá ser multiplicado pelo resultado médio final. $\mathrm{O}$ equipamento apresenta dificuldade de manuseio com grandes possibilidades de erro, necessitando de bastante atenção no momento de contornar a linha desenhada. Devido a isso, um maior número de leituras para obtenção de uma média é necessário. Deve ser considerada a necessidade da disponibilidade do aparelho e local apropriado de superfície plana e fixa para a leitura.

A pesagem dos contornos dos desenhos reproduzidos (pesagem) consistiu um método de fácil execução e relativamente rápido quando comparado aos dois anteriores. Contudo, deve-se ter atenção na padronização do local de corte das regiões a serem pesadas, igual em todos os desenhos. Há necessidade de uma balança de precisão o que encarece e que pode limitar o método.

\section{CONCLUSÕES}

Não houve diferença nos quatro métodos para determinação da AOL e AGSC. A utilização do gabarito plástico quadriculado demonstrou atender às exigências da sua utilização em pesquisa. O métodos do AutoCad, do planímetro e da pesagem dependem de equipamentos específicos e de maior custo finan- 
ceiro. A comparação entre as AOL e AGSC podem ser utilizadas como indicativo da relação carne e gordura.

\section{REFERÊNCIAS}

$\mathrm{ABCS}$. Método Brasileiro de Classificação de Carcaça. Estrela: Associação Brasileira de Criadores de Suínos, 1973. 17p (Publicação Técnica n. 2)

BALDAM, R. AutoCAD 2007: utilizando totalmente 2D, 3D e avançado, São Paulo: Imprensa, 2007.

CUNHA, E.A.; BUENO, M.S.; SANTOS, L.E.: RODA, D.S.; OTSUK, I.P. Desempenho e características de carcaça de cordeiros Suffolk alimentados com diferentes volumosos. Ciência Rural, v. 31, n. 4, p. 671-676, 2001.

CUNHA, E.A.; SANTOS, L.E.; BUENO, M.S.; RODA, D.S.; LEINZ, F.F.; RODRIGUES, C.F.C. Utilização de carneiros de raças de corte para obtenção de cordeiros precoces para abate em plantéis produtores de lã. Revista Brasileira de Zootecnia, v. 29, n. 1, p. 1590-1516, 2000.

DUTRA JR., W.M.; FERREIRA, A.S.; TAROUCO, J.U. EUCLYDES, R.F.; DONZELE, J.L.; LOPES, P.S.; CARDOSO, L.L. Estimativas de rendimentos de cortes comerciais e de tecidos de suínos em diferentes pesos de abate pela técnica de ultra-sonografia em tempo real. Revista Brasileira de Zootecnia, v. 30, n. 4, p. 1243-1250, 2001.

FIALHO, E.T.; OLIVEIRA, A.I.G.; LIMA, J.A.F.; BERTECHINI, A.G.; NASCIMENTO, J.; GOMES, M. Influência de planos de nutrição sobre as características de carcaça de suínos de diferentes genótipos abatidos entre 80 e $120 \mathrm{Kg}$. Revista Brasileira de Zootecnia, v. 27, n. 6, p. 1140-1146, 1998.

FREITAS, R.T.F.; GONÇALVES, T.M, OLIVEIRA, A.I.; FERREIRA, D.F. Avaliação de carcaças de suínos da raça Large White utilizando medidas convencionais. Revista Brasileira de Zootecnia, v. 33, n. 6, (Supl. 2), p. 20372043, 2004

GUIDONI, A.L. 2000. Melhoria de processos para a tipificação e valorização de carcaças suínas no Brasil. 2000. URL: www.cnpsa.embrapa.br/down.php?tipo=publicacoes \&cod publicacao $=290$. Consultado em 27 de junho de 2009.

JORGE.A.M.; FONTES, C.A.A.; PAULINO, M.F.; GOMES JUNIOR, P.; FERREIRA, J.N. Desempenho produtivo de animais de quatro raças zebuínas abatidos em três estágios de maturidade. 2. Característica da carcaça. Revista Brasileira de Zootecnia, v. 28, n. 2, p. 381-387, 1999.

LUCHIARI FILHO, A. Pecuária da carne bovina. 1.ed. São Paulo: Luchiari Filho, 2000. 134p.

MENEZES, L.F.O.; LOUVANDINI, H., MARTHA JÚNIOR, G.B.; McMANUS, C.; GARCIA, J.A.S.; MURATA,
L.S. Características de carcaça, componentes não-carcaça e composição tecidual e química da 12 a costela de cordeiros Santa Inês terminados em pasto com três gramíneas no período seco. Revista Brasileira de Zootecnia, v. 37, n. 7, p. 1286-1292, 2008.

OLIVEIRA, A.L.S.; DONZELE, J.L.; OLIVEIRA, R.F.M.; FERREIRA, A.S.; MOIRA, A.M.S.; GENEROSO, R.A.R. Lisina em rações para suínos machos castrados selecionados para deposição de carne. Revista Brasileira de Zootecnia, v. 32, n. 2, p. 337-343, 2003

PERKINS, T.L.; GREEN, R.D.; HAMLIN, K.E. Evaluation of ultrasonic estimates of carcass fat thickness and longissimus muscle área in beef cattle. Journal of Animal Science, v. 70, n. 4, p. 1002-1010, 1992.

PINHEIRO, M.S.; POUEY, J.L.O.F.; DEWANTIER, L.R.; GARCIA, C.A.N.; RODRIGUES, R.C.; DA SILVA, J.J.C. Avaliação de carcaça de fêmeas e machos inteiros de capivara (Hydrochaeris hydrochaeris) criados em sistema semi-intensivo. Archivos Latinoamericanos de Production Animal, v. 15, n. 2, p. 52-57, 2007.

PINHEIRO, R.M.K. Influência do grupo genético, condição sexual e uso de bioterápico, nas características de carcaça e qualidade da carne de bovinos de corte. 2007. 116f. Tese (Doutorado) - Faculdade de Veterinária, Universidade Federal Fluminense, Niterói, RJ. URL: www.uff.br/higiene_veterinaria/teses/rosangela_kalil_completa_doutorado.pdf. Consultado em 27 de junho de 2009.

RIBEIRO, F.G.; LEME, P.R.; BULLE, M.L.; DE LIMA. C.G.; DA LUZ E SILVA, S.; PEREIRA, A.S.C.; DANTE, D.P.N. Características da carcaça e qualidade da carne de tourinhos alimentados com dietas de alta energia. Revista Brasileira de Zootecnia, v. 31, n. 2, p. 749-756, 2002.

ROMA JÚNIOR, L.C. Cobertura de fibrocimento e sistema de climatização: influência no desempenho de vitelos mestiços e da raça holandesa. Faculdade de Zootecnia e Engenharia de Alimentos. 2004. 100f. Dissertação (Mestrado) - Universidade de São Paulo, Pirassununga, SP. URL: www.bv.fapesp.br/pt/dissertacoes-teses/2502/coberturafibrocimento-sistema-climatizacao-influencia/. Consultado em 20 de junho de 2009.

SAS. Statistical Analysis System. System for linear models. Cary: SAS Institutte, 1986.

SILVA, L.F.; PIRES, C.C. Avaliações quantitativas das proporções de osso, músculo e gordura da carcaça em ovinos. Revista Brasileira de Zootecnia, v. 29, n. 4, p. 1253-1260, 2000.

SIQUEIRA, E.R.; SIMÕES, C.D.; FERNANDES, S. Efeito do sexo e do peso ao abate sobre a produção de carne de cordeiros. Morfometria da carcaça, peso dos cortes, composição tecidual e componentes não constituintes da carcaça. Revista Brasileira de Zootecnia, v. 30, n. 4, p. 1299-1307, 2001. 
VELOSO, C.F.M.; LOUVANDINI, H.; KIMURA, E.A.; AZEVEDO, C.R.; ENOKI, D.R.; FRANÇA, L.D.; MCMANUS, C.M.; DELL'PORTO, A.; SANTANA, A.P. Efeitos da suplementação protéica no controle da verminose e nas características de carcaça de ovinos Santa Inês. Ciência Animal Brasileira, v. 5, n. 3, p. 131-139, 2004.

WILSON, D.E. Real-time ultrasound evaluation of de beef cattle. Study Guides. Ames: Iowa State University. I.A, 1995.

YOKOO, M.J.I.; WERNECK, J.N.;PEREIRA, M.C.; ALBUQUERQUE, L.G.; KOURY FILHO, W.; SAINZ, R.D.; LOBO, R.B.; ARAÚJO, F.R.C. Correlações genéticas entre escores visuais e características de carcaça medidas por ultrassom em bovinos de corte. Pesquisa Agropecuária Brasileira, v. 44, n. 2, p. 197-202, 2009.

Protocolado em: 11 ago. 2009 Aceito em: 02 fev.2011 